\title{
A novel design of fast and compact all-optical full- adder using nonlinear resonant cavities
}

\section{Saleh Naghizade}

Islamic Azad University Tabriz Branch

Hamed Saghaei ( $\nabla$ h.saghaei@iaushk.ac.ir)

Islamic Azad University Shahrekord Branch https://orcid.org/0000-0001-5588-3726

\section{Research Article}

Keywords: Full-adder, photonic crystal, optical Kerr effect, resonant cavity

Posted Date: February 17th, 2021

DOI: https://doi.org/10.21203/rs.3.rs-200775/v1

License: (c) (1) This work is licensed under a Creative Commons Attribution 4.0 International License. Read Full License 


\section{Abstract}

In this paper, we report a new design of all-optical full-adder using two nonlinear resonators. The PhCbased full-adder consists of three input ports (A, B, and C for input bits), two nonlinear resonant cavities, several waveguides, and two output ports (for the Sum and Carry). Eight silicon rods and a nonlinear rod composed of doped glass form each resonant cavity. The well-known plane wave expansion technique is used to calculate the photonic band structure. It shows a wide photonic bandgap in the wavelength range of $1365 \mathrm{~nm}$ to $2074 \mathrm{~nm}$ covering the $C$ and $L$ optical transmission bands. The finite-difference timedomain method is applied to study the light propagation inside the full-adder. Our numerical results demonstrate when the incoming light intensity increases, the nonlinear optical Kerr effect appears and controls the direction of light emitted inside the structure as desired. The maximum time delay and footprint of the proposed full-adder are about $3 p s$ and $758.5 \mu^{2}{ }^{2}$, respectively. Therefore, due to the low time delay and small footprint, the presented design can be used as a basic mathematical operator in the all-optical arithmetic logic unit.

\section{Introduction}

Ultrafast signal processing is a key advantage of optical devices used in telecommunication systems. Computation and communication functions must be carried out without using electrical signals in the optical domain. Signal processing is an important step in optical system design. All-optical logic gates are crucial for realizing ultrafast signal processing [1-3]. Full-adder is an optical signal process device used in every fundamental mathematical operator [4-9]. A full-adder consists of three input ports and two output ports; thus, it can add three binary digits, and two binary digits can be obtained at the output ports called Sum and Carry. A photonic crystal (PhC) may have one or more photonic band gaps (PBGs) in specific wavelength ranges in one or more directions. This unique property results in light emission in the desired directions by creating defects in the PhC [10-13]. Thus full-adder design using PhC is recommended. PhCs ring resonators (PCRRs) provide excellent performance for designing and realizing multiple optical devices with a minimal area and low manufacturing cost. Thus, they are more popular among designers. A large number of parameters, including the radius of the dielectric rod, the lattice constant, the type of rod arrangement, the dielectric constant, and the PhC unit cells' position, affect the resonant's intensity and frequency modes [14-19]. By changing every parameter, the blue or redshift occurs at resonant modes. Every PCRR has a resonant mode, acting as an optical pass-band or stopband filter. So far, a large number of PhC-based devices such as optical filters [20-23], logic gates [24$26]$, encoders [27, 28], comparators [29,30], adders and subtractors [10,31, 32], registers [33, 34], and memories [35-37], as well as all-optical clocked sequential circuits including flip-flops [38, 39], synchronous and asynchronous counters $[40,41]$ have been designed and fabricated. New functionality was created using high nonlinear dielectric rods in the resonator, and switching applications can be achieved. Recently, all-optical half-adders and full-adders were designed using linear and nonlinear properties of PhCs [42-44]. Neisy et al. [44] reported an all-optical half adder based on two nonlinear resonant cavities. These resonant cavities have different resonant modes; therefore, their coupling 
operations depend on the incoming light intensity. Banaei et al. [32] proposed an all-optical one-bit fulladder based on PCRRs. Their proposed structure was composed of two cascaded half-adders. Cheraghi et al. [6] presented an all-optical full-adder using four PCRRs. The worst cases for logics 0 and 1 were $3 \%$ and $53 \%$ for Sum port, and they were $10 \%$ and $100 \%$ for Carry port, respectively. The previous works had high input power intensities and roughly low transmission efficiency for the Sum and Carry ports. Thus we aim to improve these features.

In this paper, we present an all-optical full-adder using two nonlinear resonant cavities. The plane wave expansion (PWE) and finite-difference time-domain (FDTD) methods are used to analyze the optical behavior of the proposed structure $[45,46]$. Due to time and memory limitations, an effective refractive index method is used to reduce the 3D into 2D simulations with perfect accuracy. The paper is organized as follows. The full-adder's physical structure and the numerical results achieved by the PWE method are presented in Sect. 2. Section 3 describes the light propagation inside the full-adder using the numerical FDTD method, and the paper is closed by the conclusion in Sect. 4 .

\section{Physical Structure}

Figure 1 shows the schematic view of a typical full-adder and its truth table. We observe that a full-adder has been designed by combining two optical half-adders and an OR logic gate. Each half-adder consists of two input ports of $A$ and $B$, and two output ports of S and C that S and C stand for Sum and Carry, respectively.

The first half-adder output of $S$ has been connected to the first input port of the second half-adder. $C$ ports of optical half-adders are the OR gate's inputs and form the Carry of the final full adder and the $S$ port of the second half-adder is also the Sum port of the full-adder. Besides, $A, B$, and $C_{\text {in }}$ are the three input ports of the full-adder.

In this study, we aim to design an all-optical full-adder in a rod-based PhC. The fundamental PhC structure used to design the proposed structure consists of dielectric rods with hexagonal lattice geometry.

The refractive index and radius of dielectric rods are assumed to be 3.46 and $0.21 \mathrm{a}$, where $a$ is the lattice constant of the PhC structure. Using the PWE method, the photonic band diagram of the fundamental structure has been calculated and shown in Fig. 2 . It shows a wide PBG region at $0.27<a / \lambda<0.41$ for TM polarization mode, which is equal to $1365 \mathrm{~nm}<\lambda<2074 \mathrm{~nm}$ for $\mathrm{a}=560 \mathrm{~nm}$.

Figure 3 shows that the resonator used in the proposed full-adder consists of three waveguides (one input and two output waveguides) and two cavities. As seen in the figure, eight silicon rods (shown in red) and a nonlinear rod composed of doped glass (shown in blue in the top-right view with a radius of $128 \mathrm{~nm}$ and shown in green in the bottom-right view with a radius of $118 \mathrm{~nm}$ ) form each cavity. The doped glass has a linear refractive index of 1.4 and a nonlinear Kerr coefficient of about $10^{-14} \mathrm{~m}^{2} / \mathrm{W}$. 
An optical beam is launched in the input waveguide and dropped to one of the nonlinear cavities' output ports depending on the input power. The time-domain light propagation inside the resonator for two different optical powers are shown in Figs. 4(a), and 4(b). As shown in the figure, when an optical intensity of $10 \mathrm{~mW} / \mu \mathrm{m}^{2}$ enters the input waveguide, it exits the first output port $\left(\mathrm{O}_{1}\right)$ by creating a resonant mode in the first cavity (top) because the resonance mode is equal to the center wavelength of the input signal for this amount of optical power. When the optical intensity is $20 \mathrm{~mW} / \mu \mathrm{m}^{2}$, resonant mode occurs at the second cavity (bottom), and the optical beam goes out from the second output port $\left(\mathrm{O}_{2}\right)$.

Figure 5 shows the proposed full-adder consisting of ten waveguides and four resonant cavities (RCs) at suitable places and directions inside the fundamental PhC structure. The first half adder is formed by combining W1, W2, and W3 waveguides with RCs1. The first half adder's S and C ports are placed at the end of W4 and W5 ports, respectively. Also, W5, W6, W7, and RCs2 form the second half-adder. The outputs of W10 and W8 are its S and C ports, respectively. W4, W8, and W9 form the OR gate, and W9 works as the Carry port of the proposed full-adder. Also, the right side of the W10 works as the Sum output port. $A, B$, and $C$ are defined as the input ports of the proposed full-adder. Both RCs work with the same propagating method when the optical intensity is $10 \mathrm{~mW} / \mu \mathrm{m}^{2}$, the right-hand cavity (the one with blue rod) couples the optical beam into its output waveguide, however for the optical intensity of about $20 \mathrm{~mW} / \mu \mathrm{m}^{2}$, another cavity (the cavity with green rod) couples the optical beam to its output.

\section{Simulation Results}

We employed the FDTD method to analyze and simulate the light propagation inside the proposed fulladder shown in Fig. 5, which contains three input ports. Therefore, according to the computation principle, we have $2^{3}\left(2^{N}, N\right.$ is the number of input ports) different input states. The optical intensity of the input ports is equal to $10 \mathrm{~mW} / \mu \mathrm{m}^{2}$. The simulation results are discussed as follows for all states of the input ports.

\section{Case \#1}

In this state, all the input ports are OFF (i.e., $A=0, B=0$, and $C=0$ ); thus, there is no optical signal in the structure, and both output ports are OFF, and finally, the amounts of Sum and Carry will be zero.

\section{Case \#2}

When $A=1, B=0$, and $C=0$, the optical signal coming from input port $A$, travels close to RCs 1 through W1 and $W 3$. Since the optical intensity is equal to $10 \mathrm{~mW} / \mu \mathrm{m}^{2}$, the optical signal will be dropped into $\mathrm{W} 5$ and W7, and it is dropped into W10 using RCs2 and travels toward the full-adder's Sum port, thus, Sum = 1 and Carry $=0$. The light propagation inside the proposed full-adder is shown in Fig. 6(a). Figure 6(b) shows that in this case, the normalized powers at Sum and Carry output ports are more than $90 \%$ and less than $2 \%$, respectively. Also, the time delay is about 3.5 ps. 


\section{Case \#3}

When $A=0, B=1$, and $C=0$, the optical signal coming from input port $B$, travels close to RCs 1 through W2 and W3. Since the optical intensity is equal to $10 \mathrm{~W} / \mu \mathrm{m}^{2}$, the optical signal will be dropped into $\mathrm{W} 5$ and propagates inside W7, it is dropped into W10 using RCs2 and travels toward the Sum output port.

Therefore, we have Sum $=1$ and Carry $=0$. The light propagation inside the structure is shown in Fig. 7(a). Figure 7 (b) shows that in this case, the normalized powers at Sum and Carry are more than $90 \%$ and less than $2 \%$, respectively. Also, the time delay is about 3.5 ps.

\section{Case \#4}

When $A=0, B=0$, and $C=1$, the optical signal coming from input port $C$, travels close to RCs2 through W6 and W7. Since the optical intensity is $10 \mathrm{~mW} / \mu \mathrm{m}^{2}$, the optical signal will be dropped into $\mathrm{W} 10$ and travels toward the Sum output port; thus, we have Sum $=1$ and Carry $=0$. The light propagation inside the structure is shown in Fig. 8(a). Figure 8(b) shows that in this case, the normalized powers at Sum and Carry are more than $88 \%$ and less than $1 \%$, respectively. Also, the time delay is about 3 ps

\section{Case \#5}

When $A=1, B=1$, and $C=0$, the optical signals coming from input ports $A$ (in W1), and $B$ (in W2) are combined at $\mathrm{W} 3$ and form a resultant signal with an optical intensity of $20 \mathrm{~mW} / \mu \mathrm{m}^{2}$. Therefore, RCs 1 drops the optical signal into W4 and it travels toward the Carry output port through W9. The light propagation inside the structure is shown in Fig. 9(a). It demonstrates that there is no optical beam in W10. Thus, this case has Sum $=0$ and Carry $=1$. Figure $9(\mathrm{~b})$ shows that Sum and Carry's normalized powers are less than $5 \%$ and more than $160 \%$, respectively. Also, the rise time and the steady-state time are about 0.3 ps and $3 p s$, respectively.

\section{Case \#6}

When $A=1, B=0$, and $C=1$, the optical beam coming from input port $A$ (in W1), travels close to RCs1 through W3. since the optical intensity is $10 \mathrm{~mW} / \mu \mathrm{m}^{2}$, a resonant mode occurs, and the optical signal is dropped into W5. The optical beam coming from input port $C$ with an optical intensity of $10 \mathrm{~mW} / \mathrm{um}^{2}$ propagates in W6 and is added to the signal coming from W5 at the input of W7. Then the resultant signal is formed with an optical intensity of $20 \mathrm{~mW} / \mu \mathrm{m}^{2}$. This new signal propagates inside W7. Since the optical intensity in this waveguide is $20 \mathrm{~mW} / \mu \mathrm{m}^{2}$, the RCs2 drops the optical beam from W7 into W8, and it travels toward the Carry output port through W9. Thus, in this case, we will have Sum $=0$ and Carry $=1$. The light propagation inside the structure is shown in Fig. 10(a). Figure 10(b) shows that for this case, the normalized powers at Sum and Carry are less than $2 \%$ and more than $125 \%$, respectively. Also, the steady-state time is about 3 ps.

\section{Case \#7}


When $A=0, B=1$, and $C=1$, the optical beam coming from input port $B$ (in W2), propagates in the vicinity of the RCs1 through W3. Then the optical signal is dropped into W5 because the optical intensity is 10 $\mathrm{mW} / \mu \mathrm{m}^{2}$. Similar to Case \#6, the optical beam coming from input port $\mathrm{C}$ propagates in W6. It is added to the signal coming from W5 at the input of W7 and forms an optical beam with an intensity of $20 \mathrm{~mW} /$ $\mu \mathrm{m}^{2}$. This new signal propagates inside W7. Since the optical intensity in this waveguide is $20 \mathrm{~mW} / \mu \mathrm{m}^{2}$, the RCs2 drops the optical beam from W7 into W8, and it travels toward the Carry output port through W9. Thus we have Sum $=0$ and Carry $=1$. The light propagation inside the structure is shown in Fig. 11(a). Figure 11(b) shows that in this case, the normalized powers at Sum and Carry are less than $2 \%$ and more than $125 \%$, respectively. Also, the steady-state time is about 3 ps.

\section{Case \#8}

When $A=1, B=1$, and $C=1$, the optical signals coming from input ports $A$ (in W1), and $B$ (in W2) are combined at $\mathrm{W} 3$ and form a resultant signal with an optical intensity of $20 \mathrm{~mW} / \mu \mathrm{m}^{2}$. Therefore, RCs1 drops the optical signal into W4, and it travels toward the Carry output port through W9. The optical signal coming from input port C, travels close to RCs2 through W6 and W7. Since the optical intensity is $10 \mathrm{~mW} / \mu \mathrm{m}^{2}$, the optical signal will be dropped into $\mathrm{W} 10$ and travels toward the Sum output port; thus, we have Sum $=1$ and Carry $=1$.

The light propagation inside the structure is shown in Fig. 12(a). Figure 12(b) shows that in this case, the normalized powers at Sum and Carry are about $90 \%$ and $160 \%$, respectively. Also, the steady-state time is about 3 ps.

The numerical results of all eight input states are summarized in Table 1, and it shows that the proposed structure is acting as an all-optical full-adder. The results of this study were compared with other published papers in Table 2. It shows the input intensity, the steady-state time, and minimum output powers for logics 0 and 1 and confirms the superiority of our structure's results compared to previously reported works. 
Table 1

The outputs of the proposed full-adder for all eight input states.

\begin{tabular}{|lll|}
\hline Case & Input & Normalized Outputs (\%) \\
\cline { 2 - 3 } & A B C & Sum Carry \\
\hline$\# 1$ & 000 & 00 \\
$\# 2$ & 100 & 902 \\
$\# 3$ & 010 & 902 \\
$\# 4$ & 001 & 901 \\
$\# 5$ & 110 & 5160 \\
$\# 6$ & 101 & 2125 \\
$\# 7$ & 011 & 2125 \\
$\# 8$ & 111 & 90160 \\
\hline
\end{tabular}

Table 2

Comparison of the results of the proposed structure with previously reported works

\begin{tabular}{|llll|}
\hline Work & $\begin{array}{l}\text { Input intensity (mw/ } \\
\left.\boldsymbol{\mu m}^{2}\right)\end{array}$ & $\begin{array}{l}\text { Steady-state time } \\
(\mathbf{p s})\end{array}$ & $\begin{array}{l}\text { Minimum Transmission (\%) logic } 0 \\
\text { logic1 }\end{array}$ \\
\hline $\operatorname{Ref}[42]$ & - & 1.06 & 0.526 \\
$\operatorname{Ref}[43]$ & $4 * 10^{5}$ & 3 & 380 \\
$\operatorname{Ref}[32]$ & $5 \star 10^{5}$ & 2 & $<580$ \\
$\operatorname{Ref}[6]$ & 100 & 8 & 353 \\
This & 10 & 3 & 190 \\
Work & & & \\
\hline
\end{tabular}

\section{Conclusion}

In summary, we designed a fast and compact all-optical full-adder using several nonlinear nanocavities. Eight different states for three input digits were simulated using the well-known FDTD method assuming PML boundary conditions. The numerical results revealed the proposed full-adder has a maximum steady-state time of about $3 \mathrm{ps}$. The structure's total size was equal to $758.5 \mu \mathrm{m}^{2}$, which was more compact than other works. Furthermore, appropriate power margins for logics zero and one were 
obtained at $1 \%$ and $90 \%$, respectively. As a result, the presented half-adder can be used in optical integrated circuits for high-speed signal processing.

\section{Declarations}

\section{Compliance with ethical standards}

Confict of interest The authors declare that they have no known competing fnancial interests or personal relationships that could have appeared to infuence the work reported in this paper.

\section{References}

1. H. Sharifi, S. M. Hamidi, and K. Navi, "A new design procedure for all-optical photonic crystal logic gates and functions based on threshold logic," Optics Communications 370, 231-238 (2016).

2. A. Rahmani and F. Mehdizadeh, "Application of nonlinear PhCRRs in realizing all optical half-adder," Optical and Quantum Electronics 50(1), 30 (2018).

3. H. Saghaei, A. Zahedi, R. Karimzadeh, and F. Parandin, "Line defects on photonic crystals for the design of all-optical power splitters and digital logic gates," Superlattices and Microstructures 110, 133-138 (2017).

4. M. J. Maleki, A. Mir, and M. Soroosh, "Designing an ultra-fast all-optical full-adder based on nonlinear photonic crystal cavities," Optical and Quantum Electronics 52(4), 1-11 (2020).

5. A. M. Vali-Nasab, A. Mir, and R. Talebzadeh, "Design and simulation of an all optical full-adder based on photonic crystals," Optical and Quantum Electronics 51(5), (2019).

6. F. Cheraghi, M. Soroosh, and G. Akbarizadeh, "An ultra-compact all optical full adder based on nonlinear photonic crystal resonant cavities," Superlattices and Microstructures 113, 359-365 (2018).

7. Q. Liu and Z. B. Ouyang, "All-optical half adder based on cross structures in two-dimensional photonic crystals," Guangzi Xuebao/Acta Photonica Sinica 37(SUPPL. 2), 46-50 (2008).

8. Y. C. Jiang, S. Bin Liu, H. F. Zhang, and X. K. Kong, "Realization of all optical half-adder based on selfcollimated beams by two-dimensional photonic crystals," Optics Communications 348, 90-94 (2015).

9. M. H. Sani, A. A. Tabrizi, H. Saghaei, and R. Karimzadeh, "An ultrafast all-optical half adder using nonlinear ring resonators in photonic crystal microstructure," Optical and Quantum Electronics 52(2), 107 (2020).

10. M. Hosseinzadeh Sani, A. Ghanbari, and H. Saghaei, "An ultra-narrowband all-optical filter based on the resonant cavities in rod-based photonic crystal microstructure," Optical and Quantum Electronics 52(6), 295 (2020).

11. M. Hosseinzadeh Sani, H. Saghaei, M. A. Mehranpour, and A. Asgariyan Tabrizi, "A Novel All-Optical Sensor Design Based on a Tunable Resonant Nanocavity in Photonic Crystal Microstructure 
Applicable in MEMS Accelerometers," Photonic Sensors (2020).

12. F. Mehdizadeh, M. Soroosh, H. Alipour-Banaei, and E. Farshidi, "A novel proposal for all optical analog-to-digital converter based on photonic crystal structures," IEEE Photonics Journal 9(2), 1-11 (2017).

13. H. Alipour-Banaei and F. Mehdizadeh, "Bandgap calculation of 2D hexagonal photonic crystal structures based on regression analysis," Journal of Optical Communications 34(4), 285-293 (2013).

14. A. Tavousi, M. A. Mansouri-Birjandi, M. Ghadrdan, and M. Ranjbar-Torkamani, "Application of photonic crystal ring resonator nonlinear response for full-optical tunable add-drop filtering," Photonic Network Communications 34(1), 131-139 (2017).

15. M. A. Mansouri-Birjandi, A. Tavousi, and M. Ghadrdan, "Full-optical tunable add/drop filter based on nonlinear photonic crystal ring resonators," Photonics and Nanostructures - Fundamentals and Applications 21, 44-51 (2016).

16. U. Biswas, J. K. Rakshit, and G. K. Bharti, "Design of photonic crystal microring resonator based alloptical refractive-index sensor for analyzing different milk constituents," Optical and Quantum Electronics 52(1), 19 (2020).

17. A. Farmani, M. Soroosh, M. H. Mozaffari, and T. Daghooghi, "Optical nanosensors for cancer and virus detections," in Nanosensors for Smart Cities (Elsevier, 2020), pp. 419-432.

18. S. Naghizade, S. Mohammadi, and H. Khoshsima, "Design and simulation of an all optical 8 to 3 binary encoder based on optimized photonic crystal or gates," Journal of Optical Communications 410, 793-798 (2018).

19. S. Naghizade and S. M. Sattari-Esfahlan, "Loss-less elliptical channel drop filter for WDM applications," Journal of Optical Communications 40(4), 379-384 (2017).

20. Y. Guo, S. Zhang, J. Li, S. Li, and T. Cheng, "A sensor-compatible polarization filter based on photonic crystal fiber with dual-open-ring channel by surface plasmon resonance," Optik 193, 162868 (2019).

21. H. Alipour-Banaei, M. Jahanara, and F. Mehdizadeh, "T-shaped channel drop filter based on photonic crystal ring resonator," Optik 125(18), 5348-5351 (2014).

22. M. R. Rakhshani and M. A. Mansouri-Birjandi, "Realization of tunable optical filter by photonic crystal ring resonators," Optik 124(22), 5377-5380 (2013).

23. S. Naghizade and H. Saghaei, "Tunable graphene-on-insulator band-stop filter at the mid-infrared region," Optical and Quantum Electronics 52(4), 224 (2020).

24. R. M. Younis, N. F. F. Areed, and S. S. A. Obayya, "Fully integrated and and or optical logic gates," IEEE Photonics Technology Letters 26(19), 1900-1903 (2014).

25. P. Andalib and N. Granpayeh, "All-optical ultracompact photonic crystal AND gate based on nonlinear ring resonators," Journal of the Optical Society of America B 26(1), 10 (2009).

26. H. M. E. Hussein, T. A. Ali, and N. H. Rafat, "New designs of a complete set of Photonic Crystals logic gates," Optics Communications 411, 175-181 (2018). 
27. S. Naghizade and H. Khoshsima, "Low input power an all optical $4 \times 2$ encoder based on triangular lattice shape photonic crystal," Journal of Optical Communications 1, 1-8 (2018).

28. T. A. Moniem, "All-optical digital $4 \times 2$ encoder based on 2D photonic crystal ring resonators," Journal of Modern Optics 63(8), 735-741 (2016).

29. V. Fakouri-Farid and A. Andalib, "Design and simulation of an all optical photonic crystal-based comparator," Optik 172, 241-248 (2018).

30. H. Jile, "Realization of an all-optical comparator using beam interference inside photonic crystal waveguides," Applied Optics 59(12), 3714 (2020).

31. R. Moradi, "All optical half subtractor using photonic crystal based nonlinear ring resonators," Optical and Quantum Electronics 51(4), 119 (2019).

32. H. Alipour-Banaei and H. Seif-Dargahi, "Photonic crystal based 1-bit full-adder optical circuit by using ring resonators in a nonlinear structure," Photonics and Nanostructures-Fundamentals and Applications 24, 29-34 (2017).

33. N. Pahari and A. Guchhait, "All-optical Serial Data Transfer between Registers using optical non-linear materials," Optik 123(5), 462-466 (2012).

34. M. Martinez-Dorantes, W. Alt, J. Gallego, S. Ghosh, L. Ratschbacher, Y. Völzke, and D. Meschede, "Fast Nondestructive Parallel Readout of Neutral Atom Registers in Optical Potentials," Physical Review Letters 119(18), 180503 (2017).

35. E. Kuramochi, K. Nozaki, A. Shinya, K. Takeda, T. Sato, S. Matsuo, H. Taniyama, H. Sumikura, and M. Notomi, "Large-scale integration of wavelength-addressable all-optical memories on a photonic crystal chip," Nature Photonics 8(6), 474-481 (2014).

36. T. Alexoudi, G. T. Kanellos, and N. Pleros, "Optical RAM and integrated optical memories: a survey," Light: Science and Applications 9(1), 1-16 (2020).

37. T. Uda, A. Ishii, and Y. K. Kato, "Single Carbon Nanotubes as Ultrasmall All-Optical Memories," ACS Photonics 5(2), 559-565 (2018).

38. R. Kumar, K. Huybrechts, L. Liu, T. Spuessens, G. Roelkens, E. J. Geluk, T. De Vries, P. Regreny, D. Van Thourhout, R. Baets, and G. Morthier, "An ultra-small, low-power all-optical flip-flop memory on a silicon chip," Optics InfoBase Conference Papers 4(3), 182-187 (2010).

39. P. Sethi and S. Roy, "Ultrafast All-Optical Flip-Flops, Simultaneous Comparator-Decoder and Reconfigurable Logic Unit with Silicon Microring Resonator Switches," IEEE Journal on Selected Topics in Quantum Electronics 20(4), 118-125 (2014).

40. A. Poustie, R. J. Manning, A. E. Kelly, and K. J. Blow, "All-optical binary counter," Optics Express 6(3), $69(2000)$.

41. S. Kaur and R. S. Kaler, " $5 \mathrm{GHz}$ all-optical binary counter employing SOA-MZIs and an optical NOT gate," Journal of Optics (United Kingdom) 16(3), 35201 (2014).

42. S. Swarnakar, S. Kumar, and S. Sharma, "Performance analysis of all-optical full-adder based on twodimensional photonic crystals," Journal of Computational Electronics 17(3), 1124-1134 (2018). 
43. M. Moradi, M. Danaie, and A. A. Orouji, "Design and analysis of an optical full-adder based on nonlinear photonic crystal ring resonators," Optik 172, 127-136 (2018).

44. M. Neisy, M. Soroosh, and K. Ansari-Asl, "All optical half adder based on photonic crystal resonant cavities," Photonic Network Communications 35(2), 245-250 (2018).

45. S. Johnson and J. Joannopoulos, "Block-iterative frequency-domain methods for Maxwell's equations in a planewave basis," Optics Express 8(3), 173 (2001).

46. M. Qiu, "Effective index method for heterostructure-slab-waveguide-based two-dimensional photonic crystals," Applied Physics Letters 81(7), 1163-1165 (2002).

\section{Figures}

(b)

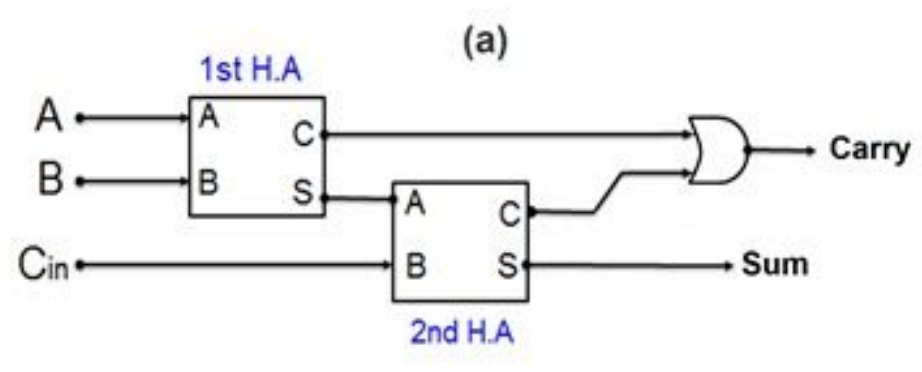

\begin{tabular}{|c|c|c|c|c|}
\hline \multicolumn{3}{|c|}{ Input } & \multicolumn{2}{c|}{ Output } \\
\hline A & B & Cin & Sum & Carry \\
\hline 0 & 0 & 0 & 0 & 0 \\
\hline 0 & 0 & 1 & 1 & 0 \\
\hline 0 & 1 & 0 & 1 & 0 \\
\hline 0 & 1 & 1 & 0 & 1 \\
\hline 1 & 0 & 0 & 1 & 0 \\
\hline 1 & 0 & 1 & 0 & 1 \\
\hline 1 & 1 & 0 & 0 & 1 \\
\hline 1 & 1 & 1 & 1 & 1 \\
\hline
\end{tabular}

\section{Figure 1}

Illustration of (a) the full-adder circuit consisting of two half-adders and an OR logic gate, three input ports of $A, B$, and Cin, and two output ports of Sum and Carry, (b) the truth table of full-adder for all states. 


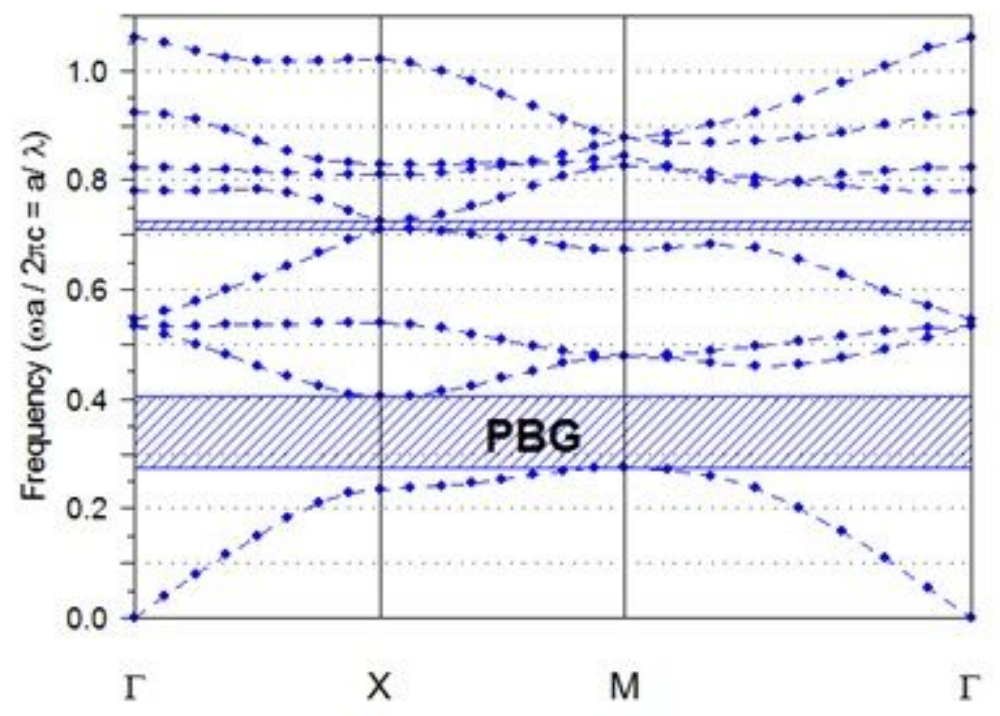

Figure 2

Photonic band diagram of the fundamental rod-based PhC for the TM polarization mode.

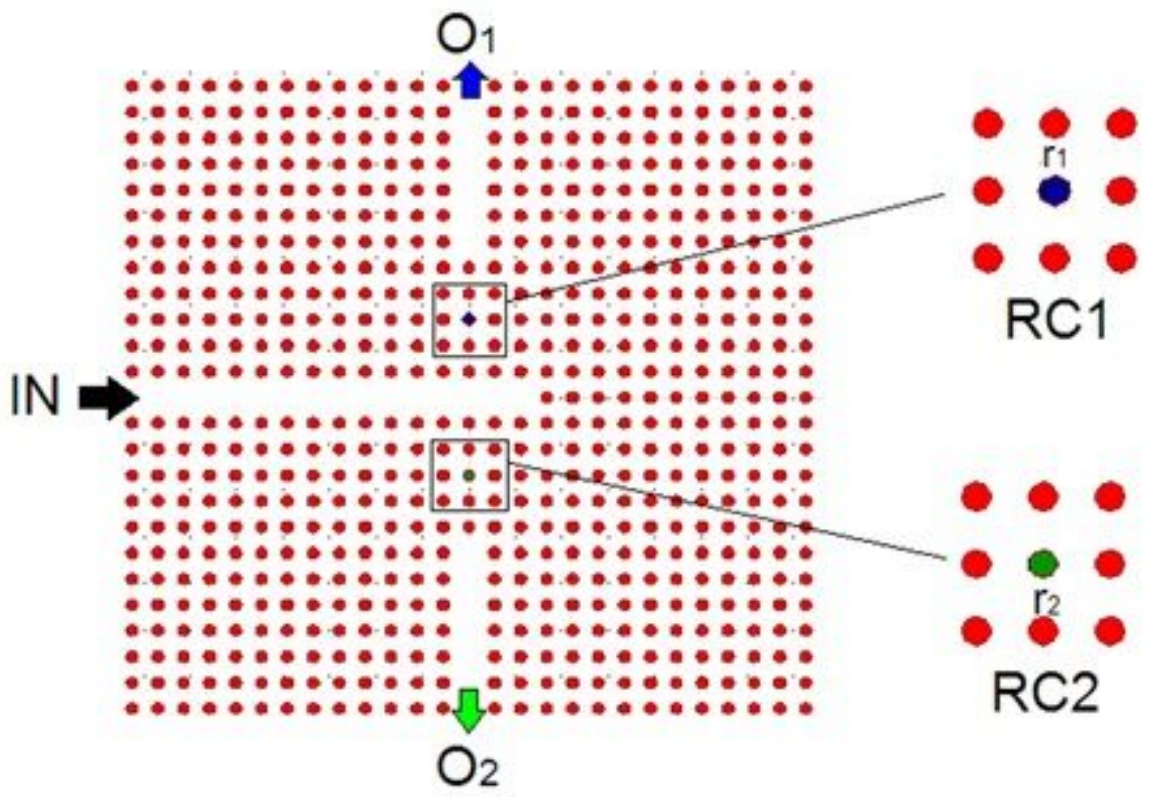

Figure 3

The schematic view of $\mathrm{PhC}$ resonator consisting of three waveguides (one input and two output waveguides) and two resonant cavities. 


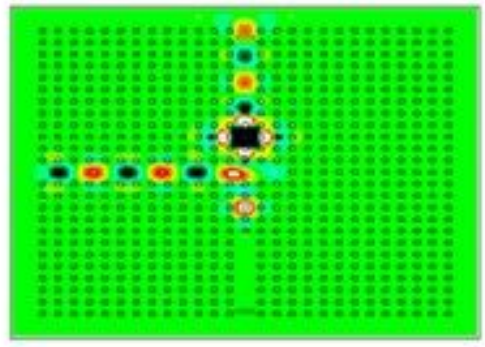

(a)

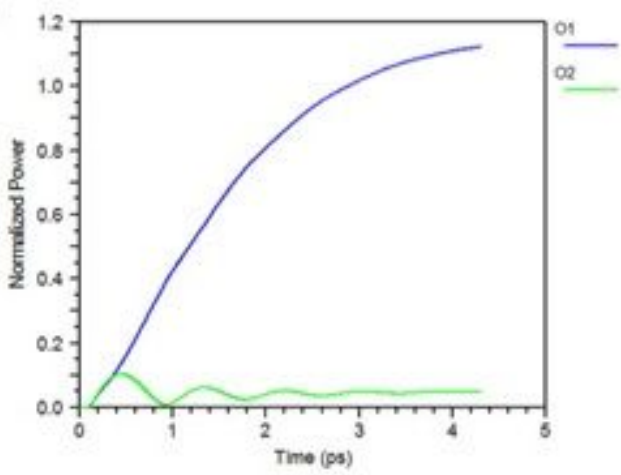

(b)
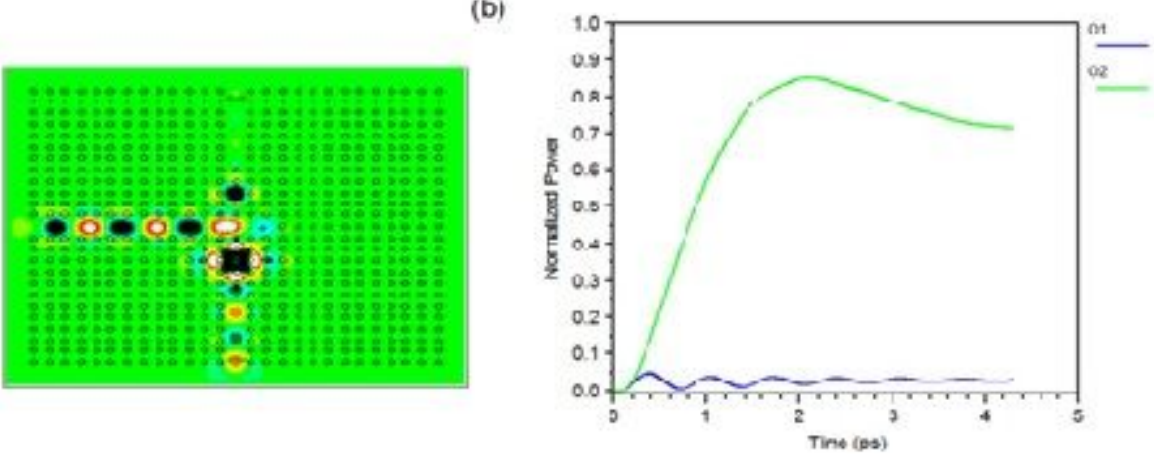

Figure 4

Light propagation inside the proposed resonator for different input optical intensities of (a) $10 \mathrm{~mW} / \mu \mathrm{m} 2$ and (b) $20 \mathrm{~mW} / \mu \mathrm{m} 2$.

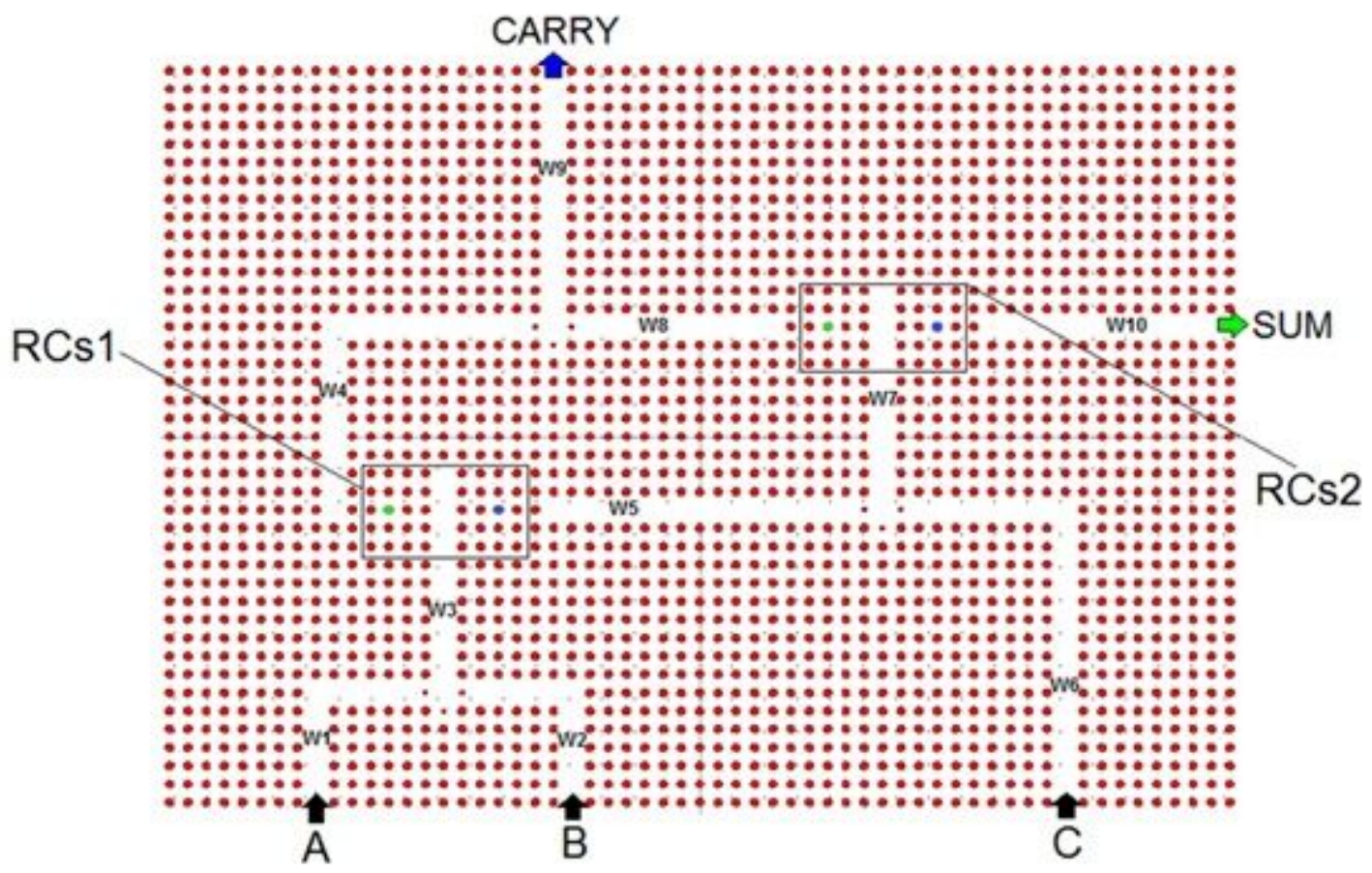




\section{Figure 5}

The schematic view the proposed all-optical full-adder consisting of three input ports (for three input digits), ten waveguides, four resonant cavities, and two output ports (for Sum and Carry).

(a)

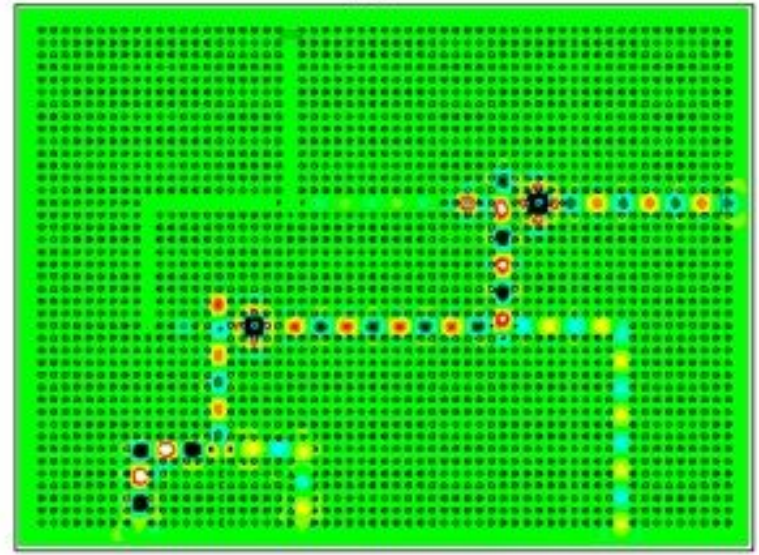

(bi)

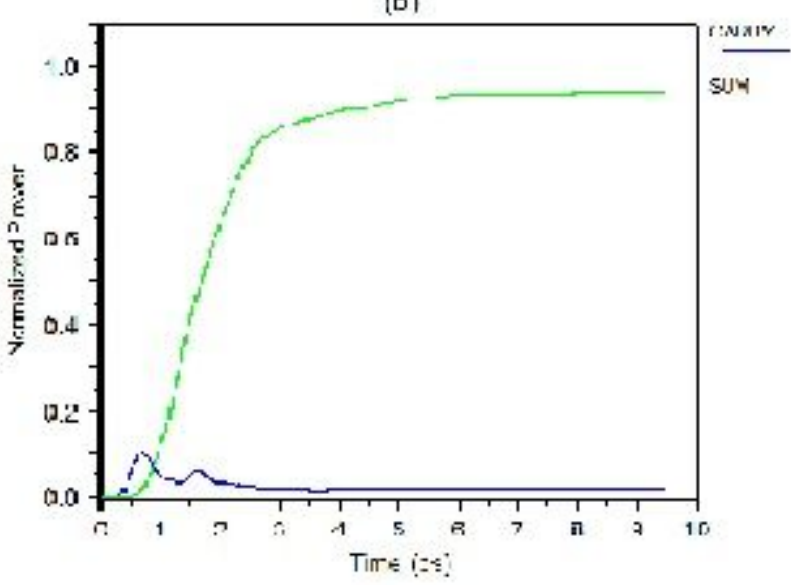

\section{Figure 6}

Illustration of (a) light propagation and (b) output powers of the proposed full-adder for Case \#2.

(a)

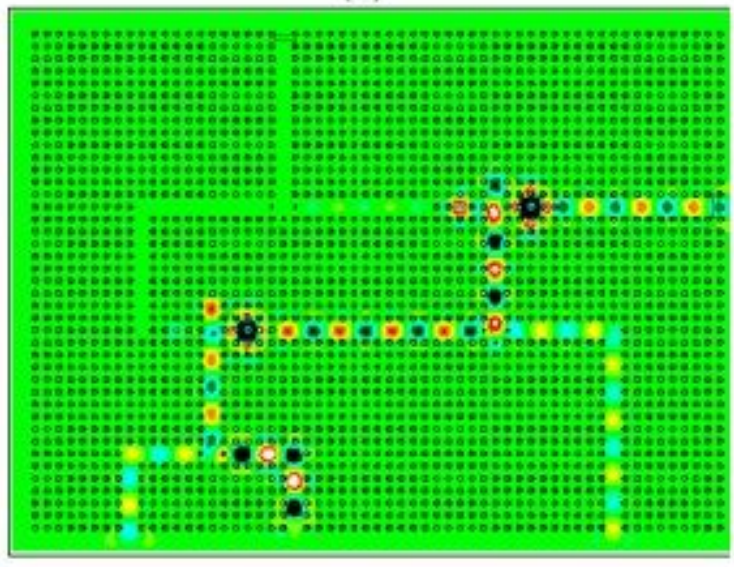

(b)

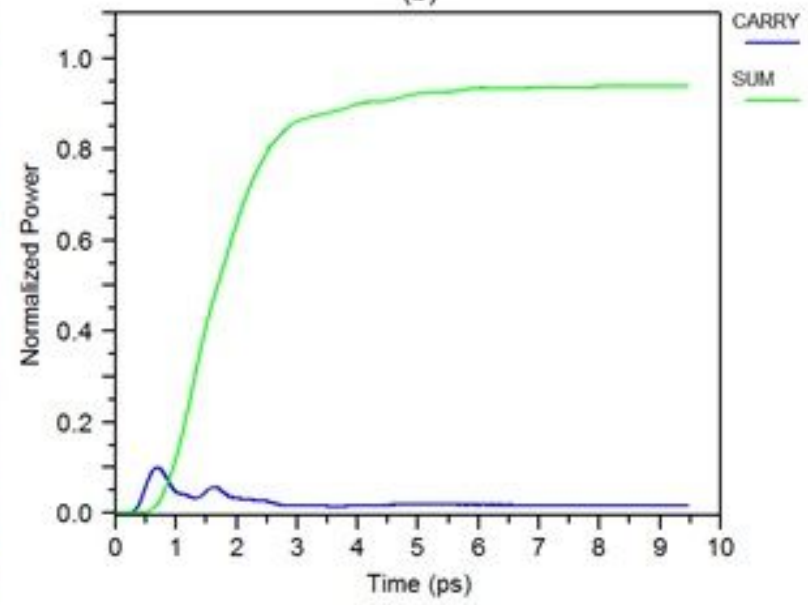

Figure 7

Illustration of (a) light propagation and (b) output powers of the proposed full-adder for Case \#3. 
(a)

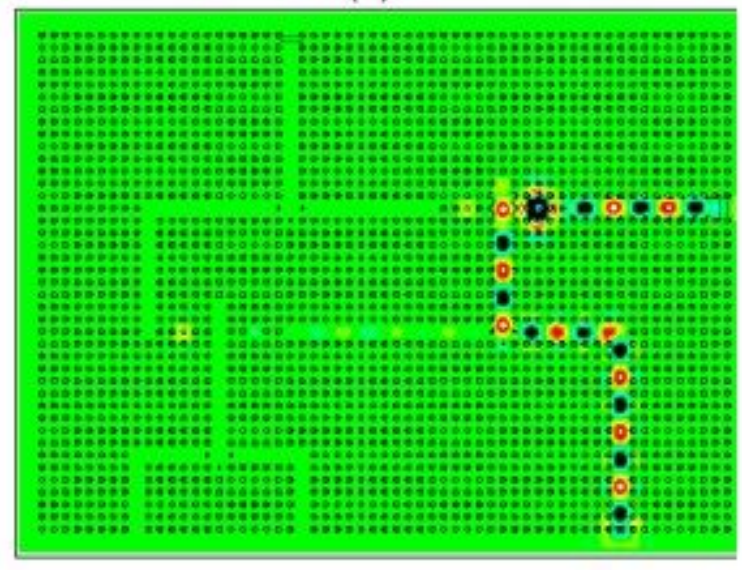

(b)

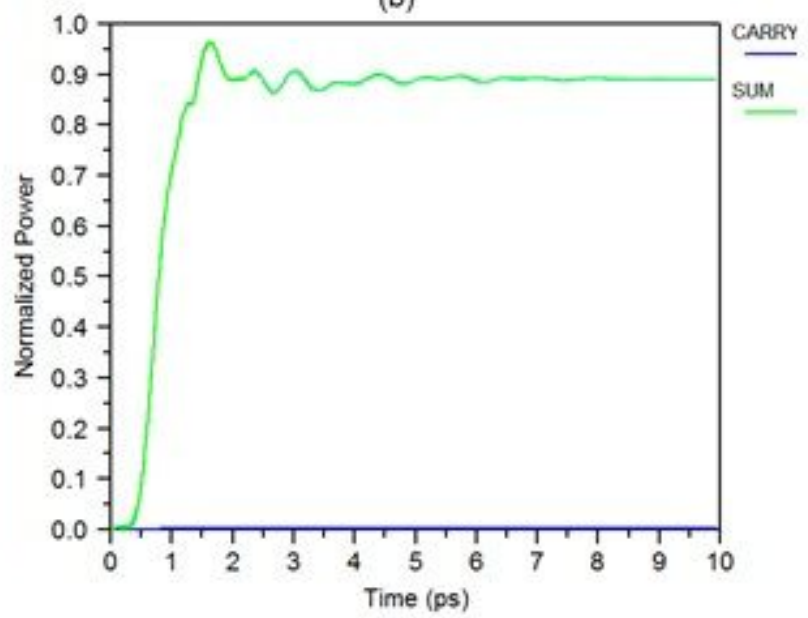

Figure 8

Illustration of (a) light propagation and (b) output powers of the proposed full-adder for Case \#4.

(a)
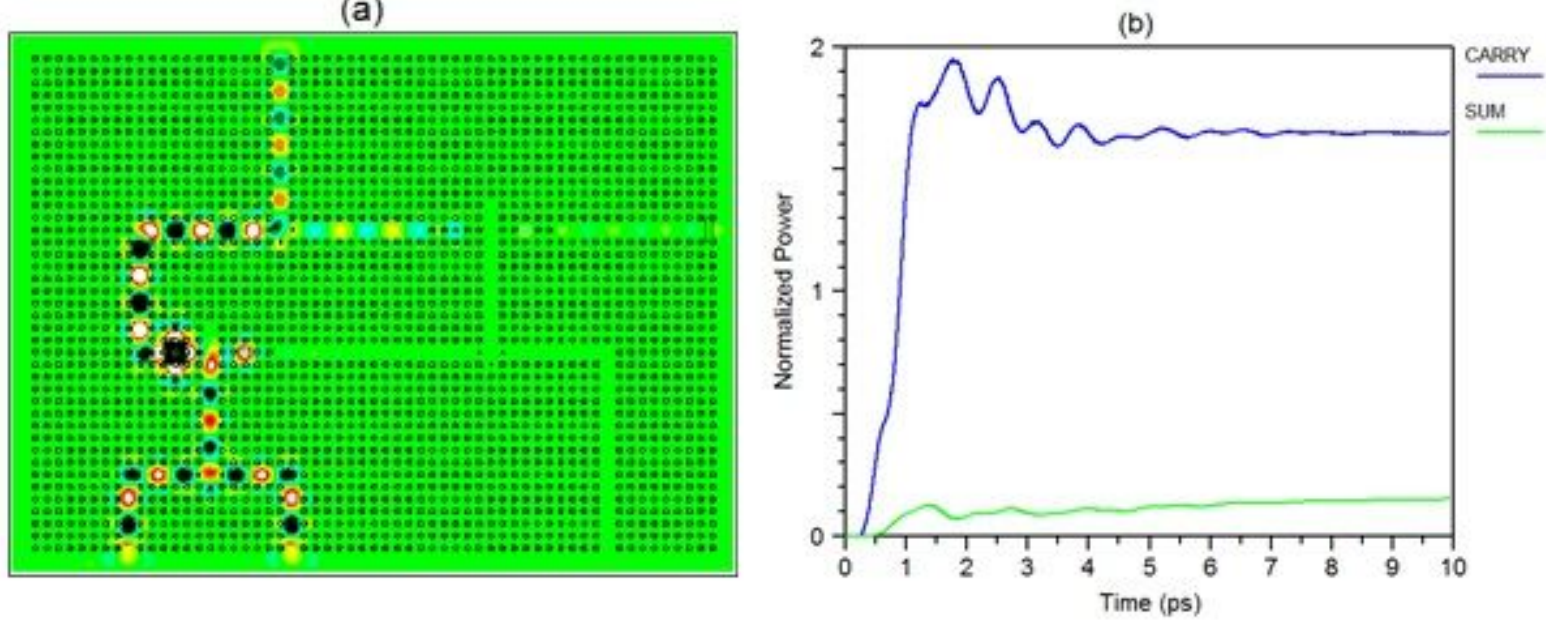

\section{Figure 9}

Illustration of (a) light propagation and (b) output powers of the proposed full-adder for Case \#5.

(a)

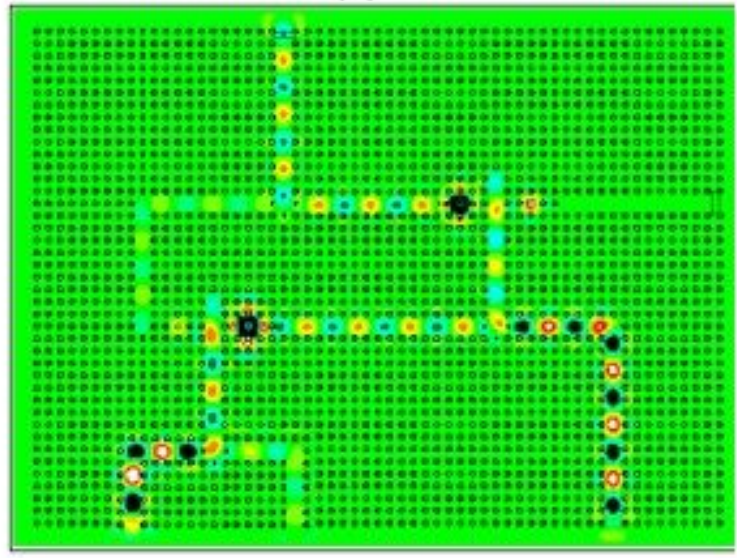

(b)

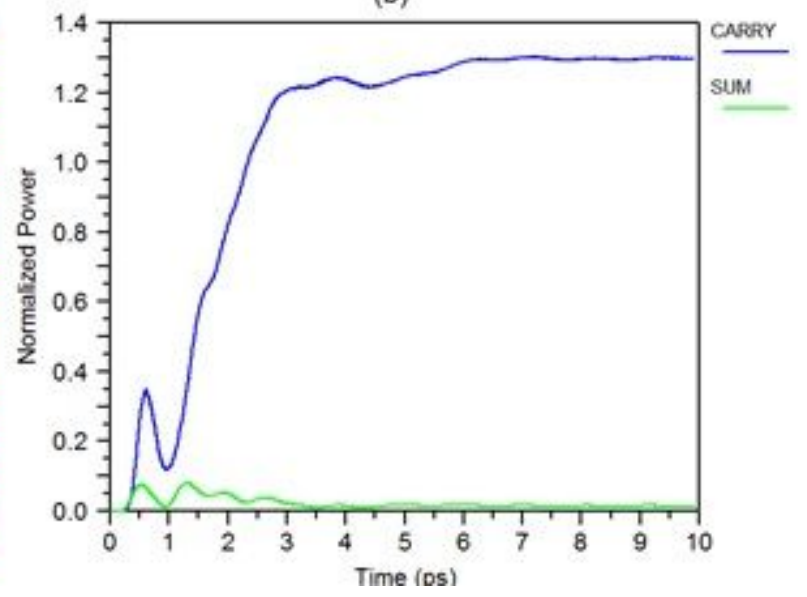


Figure 10

Illustration of (a) light propagation and (b) output powers of the proposed full-adder for Case \#6.

(a)

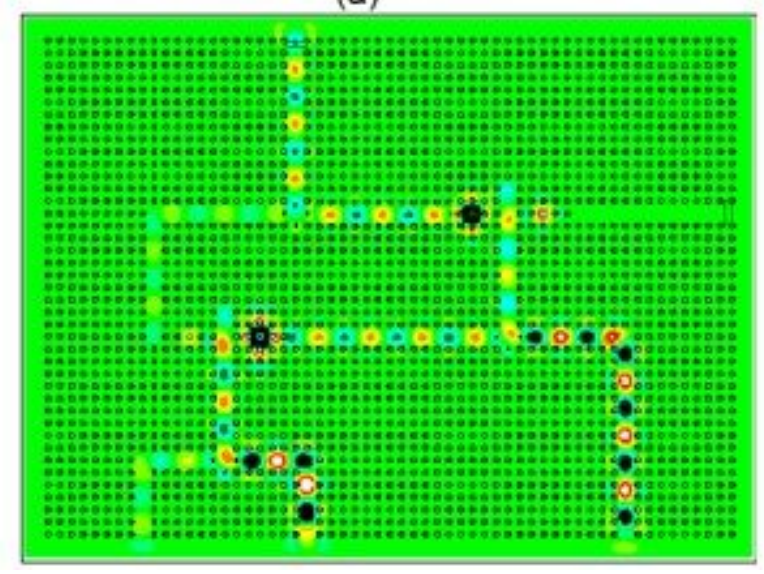

(b)

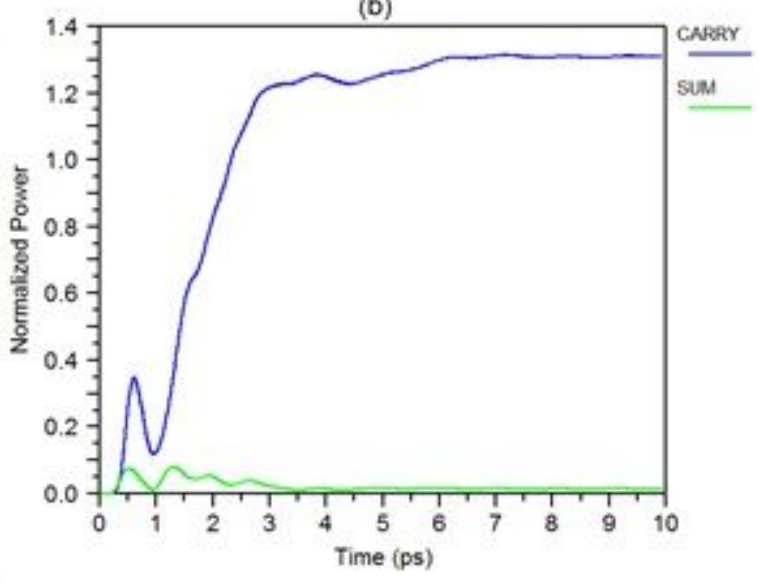

\section{Figure 11}

Illustration of (a) light propagation and (b) output powers of the proposed full-adder for Case \#7.

(a)

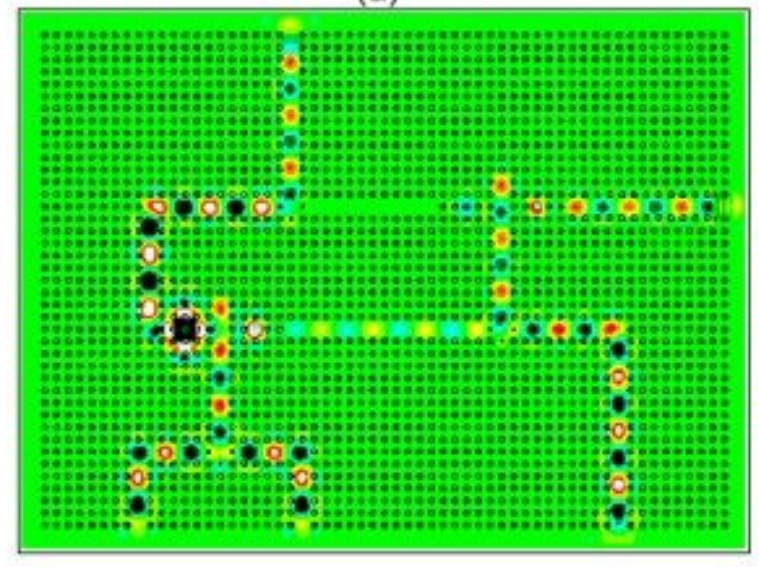

(b)

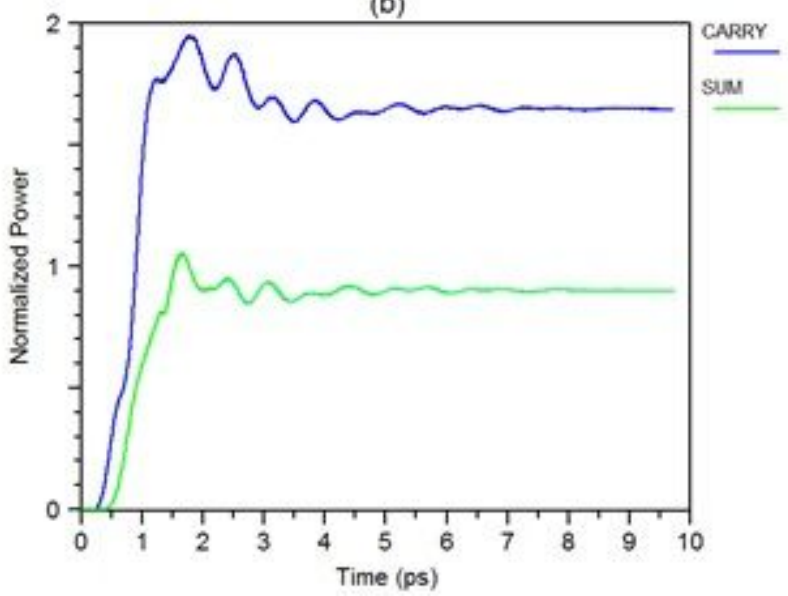

Figure 12

Illustration of (a) light propagation and (b) output powers of the proposed full-adder for Case \#8. 